\title{
TOTAL EXPOSURE HEALTH
}

\author{
Richard Hartman ${ }^{1}$ and Mark Oxley ${ }^{2}$ \\ Air Force Institute of Technology \\ WPAFB OH 45433, USA
}

\begin{abstract}
This paper will introduce the origins and demonstrate how the concept and implementation of Total Exposure Health (TEH) is ushering in a bold solution to capture workplace, environmental, and lifestyle exposures to the individual using advances in science, technology, and informatics.

It will also introduce and describe the power behind Total Exposure Health, which is a mathematical process to quantitatively evaluate individual health risks based on genetics, occupational, lifestyle, and environmental exposures, medical disposition, protective factors, etc., forming the Individual Exposure Health Risk Profile (IEHRP).
\end{abstract}

\section{KEYWORDS}

Genomics, Informatics, Noise, Precision Health, Risk Assessment, Sensors

\section{INTRODUCTION}

After the military's experience of health consequences relating to Agent Orange exposure during the Vietnam War and the significant adverse health claims by Service members deployed to the Persian Gulf War, the necessity to monitor and document exposures was codified into Public Law in 1997 whereupon the Department of Defense (DoD) was mandated to develop a deployment health surveillance system to detect, document, prevent, or minimize health problems arising as a result of occupational and environmental exposures during deployments and operations.

To respond to the aforementioned mandates and improve the long-term health and welfare of the warfighter, the Department of Defense (DoD) created the Total Exposure Health (TEH) Initiative, an innovative approach to protect its military members and beneficiaries, promote readiness, and deliver personalized risk information in support of enhanced primary care (Figure 1).

\footnotetext{
${ }^{1}$ Richard Hartman, $\mathrm{PhD}$, is an independent consultant for the Air Force Institute of Technology.

${ }^{2}$ Mark Oxley, $\mathrm{PhD}$, is a full professor of Mathematics in the Department of Mathematics and Statistics, in the Graduate School of Engineering and Management at the Air Force Institute of Technology, Wright-Patterson Air Force Base, Ohio.
} 


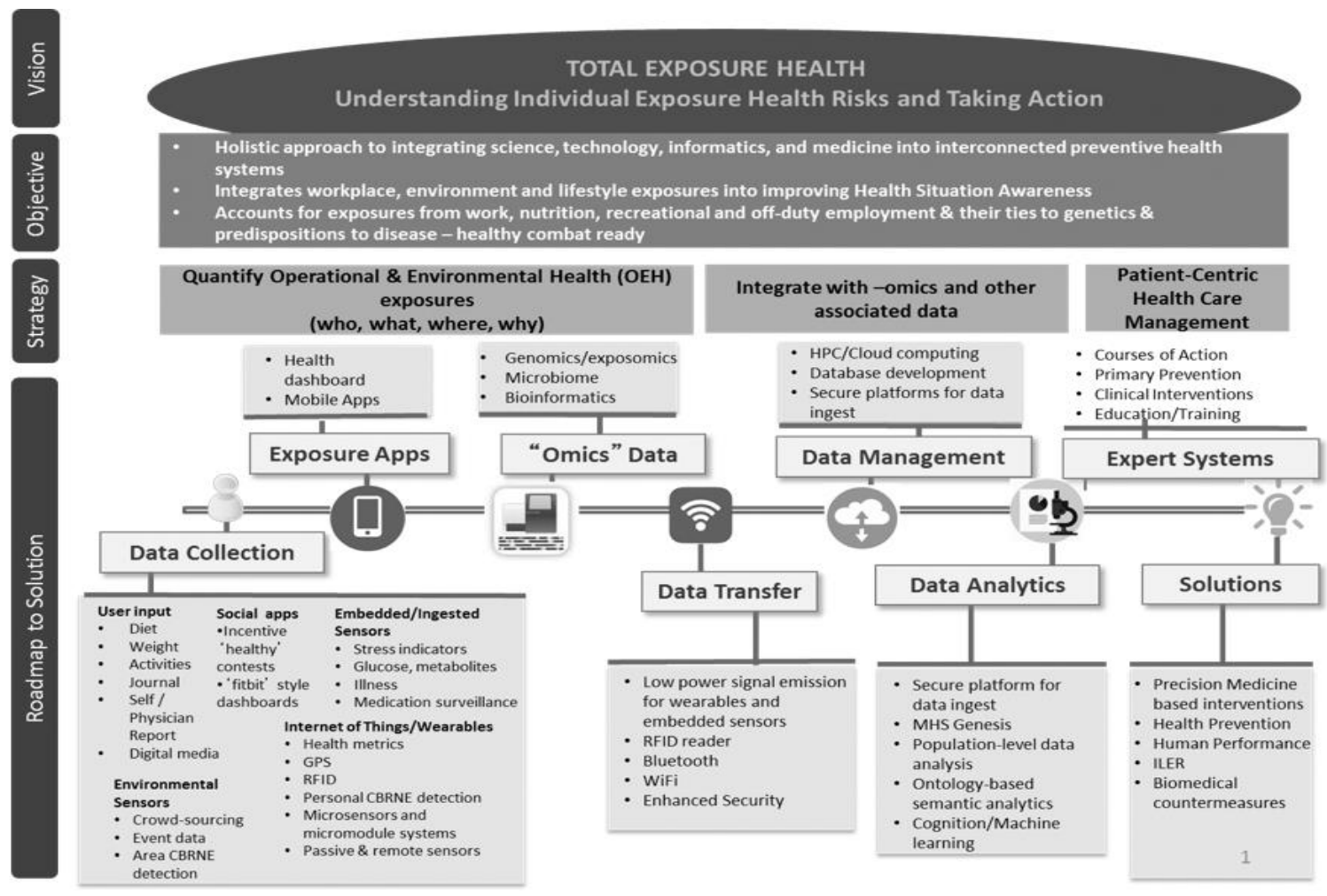

Figure 1. TEH end-to-end system development

\section{TOTAL EXPOSURE HEALTH (TEH)}

While the DoD made advances in health surveillance, the issues to collect, document, and act upon occupational and environmental health exposures while deployed remains a challenge. This is due to the nature of deployments, which are $24 / 7$ workplaces, so the need to develop a more comprehensive view of what exposure meant beyond the typical 40-hr workweek and workplace was necessary. This meant expanding the practice of monitoring occupational and environmental exposures to include lifestyle choices.

This new way of characterizing worker well-being originated at the National Institute for Occupational Safety and Health (NIOSH) through Total Worker Health (TWH) as "a holistic approach to worker well-being that acknowledges risk factors related to work that contribute to health problems previously considered unrelated to work." (NIOSH, 2015)

The DoD recognized the validity of this approach but sought to realize a greater level of personalization by utilizing recent advances in the exposure sciences, genomics, sensor and data technologies, and health informatics which would aid in a more complete understanding of an individual's health risks, root causes of disease and injury, and innovative but accessible methods for primary prevention. The result is what is now referred to as Total Exposure Health (TEH).

Operationalizing TEH required partnering across multiple program areas, using traditional and emerging exposure assessment technologies (including sensors and-omics-based molecular biology), as well as leveraging a Big Data infrastructure and advanced analytics.

While the near-term goal of TEH is to improve exposure characterization and understand individual variability, susceptibility, and vulnerability to cumulative exposures and risks factors, the first achievement was to demonstrate the feasibility of TEH and the ability to collect exposure data 24/7 to better understand the effects of total exposure. In the long-term, TEH adds genomics and other data sets to translate these findings into clinically actionable recommendations for health prevention to improve both the health and well-being of both the workforce and also their beneficiaries. 
Though the ultimate implementation of TEH will monitor a wide range of physical risks such as radiation and chemical exposure from the workplace and environment as well as lifestyle risks from recreational activities and diet, the first focus was on noise exposure.

\subsection{Noise Exposure Demonstration Project (NEDP)}

Noise was the focus for this demonstration project because it is: (1) an exposure that does not discriminate by age, race, gender, and/or socioeconomic status; (2) costly (currently hearing health expenditures range from $\$ 1.5$ billion to $\$ 2$ billion annually for the U.S. Department of Veterans Affairs in benefits and medical costs) (Alamgir, et al, 2016) and; (3) affects approximately 10\% of the U.S. population according to a 2017 report by the Centers for Disease Control and Prevention, where 40 million American adults show signs of noise-induced hearing loss (NIHL). (Carroll, et al, 2017).

NEDP used sensor technology to collect total noise exposure $24 \mathrm{hr}$ a day, seven days a week. Unique to our noise monitoring devices, the wearable sensor collects sound events from both ambient noise in the workplace and environment as well as in-ear sounds from ear buds used with smartphones and other devices playing digital media (such as music) with a smartphone app recording those decibel (dB) readings.

Nineteen subjects participated in this study at Moody Air Force Base, Georgia over 10 days in June 2018. We captured 12,680 noise events of $70 \mathrm{dBA}$ or above, with 2,968 events, or $23 \%$, were $95 \mathrm{dBA}$ or above. (A-weighted decibels are adjusted for human hearing.) The average daily (24-hr) decibel dose was about 75 $\mathrm{dBA}$. About $10 \%$ of the subjects had total daily noise exposures under $70 \mathrm{~dB}$, and 10 percent over $80 \mathrm{~dB}$, with the majority in the middle.

We found high noise exposure both at the workplace (46\%) and off-duty (52\%), and significant cumulative high noise individual exposure (three to $27 \mathrm{hr}$ over the 10-day study period). We also identified geospatial "hotspot" locations of exposure across the population.

Overall, the NDEP met its objectives as a successful TEH demonstration project. We developed a low-cost noise dosimeter/sensor that monitors external and media smartphone device noise sound levels around the clock; used advanced analytics to collate multiple sensor devices, with geospatial layering; and managed participant compliance.

Recognizing that development of NIHL varies among populations who have been exposed to the same levels of noise, we wondered if there were genetic markers associated with a predisposition. We identified ten published studies, with small to modest sample sizes, which have indeed established a link between multiple genetic variants associated with NIHL. The effect is surprisingly large, with odds ratios of 5.2 to 22.36 indicating an elevated risk. (PLOS Onc, 2015).

Furthermore, within the broader USAF population, of which the NDEP participants are a subset, $17 \%$ of the 2,000 who have had their genes fully sequenced showed a particular gene variant, rs7598759, indicating they may be at a substantially increased risk for a hearing threshold shift - that is, of a hearing loss susceptibility due to noise.

This finding led to a new question: What if we could take a person's cumulative, daily exposure to noise and combine this data with their genetic predisposition for NIHL to optimally protect against it? More broadly speaking, what if we could predict, by aggregating big data and genomics, a person's susceptibility to external health risk factors - and take action to alleviate it using individualized health protocols?

We determined that by using an individual's medical records we could identify pre-existing conditions that would also increase the risk of NIHL, thus bringing together exposure data, genomics data and medical record data together to describe a more holistic montage of the persons risk to a particular exposure.

This estimate/calculation of individual health risk factors involved the need for a mathematical/informatics process that could merge and analyze various data from sensors, medical records, and unstructured information as well as genomics data to identify relationships between them at the individual level called the Individual Exposure Health Risk Profile (IEHRP).

\subsection{Individual Exposure Health Risk Profile (IEHRP)}

The IEHRP integrates exposure data from both traditional and cutting edge emerging exposure assessment technologies (including sensors and "-omics" based molecular biology) with clinical and genomic data. Combining the three: 1) the exposure measurement, 2) the genetic proclivity associated with the exposure 
and 3) current clinical history associated with the exposure, provides a better description of the individual's risk than would a focus on any one variable. This combination originally resulted in the Individual Exposure Health Risk Index (IEHRI) defined by

$$
\operatorname{IEHRI}_{1}=\mathrm{v}_{1,1}+\mathrm{v}_{1,2}+\mathrm{v}_{1,3}
$$

where $\mathrm{v}_{1,1}$ is the exposure measure, the $\mathrm{v}_{1,2}$ is genetic proclivity to the exposure and $\mathrm{v}_{1,3}$ is the clinical effect per the evidence in the individual's medical record (medical history). For example, when evaluating noise, the IEHRI becomes:

$$
\operatorname{IEHRI}_{(\text {Noise })}=\mathrm{v}_{(\text {Noise, measure })}+\mathrm{v}_{(\text {Noise, genes })}+\mathrm{v}_{(\text {Noise, history })}
$$

Accounting for multiple exposures lead to the development of the IEHRP, which combines multiple IEHRI's for an individual, represented by the equation (3) for (i) exposures.

$$
\begin{aligned}
& \text { IEHRP }\left(\text { IEHRI }_{1}, \text { IEHRI }_{2}, \ldots, \text { IEHRI }_{i}\right)=\left(\left[\mathrm{v}_{1,1}+\mathrm{v}_{1,2}+\mathrm{v}_{1,3}\right],\left[\mathrm{v}_{2,1}+\mathrm{v}_{2,2}+\mathrm{v}_{2,3}\right], \ldots,\left[\mathrm{v}_{\mathrm{i}, 1}+\mathrm{v}_{\mathrm{i}, 2}+\right.\right. \\
& \left.\mathrm{V}_{\mathrm{i}, 3}\right] \text { ) }
\end{aligned}
$$

With the IEHRP equipped to address multiple exposures, the limitations to the IEHRI became evident, which only accounted for three variables to describe an individual exposure. This was observed early in the

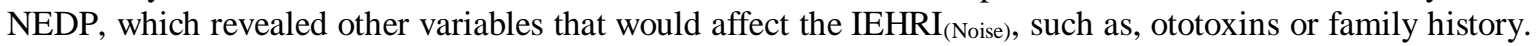
This required the IEHRP to be modified so it could account for multiple confounding factors, and is represented by the equation (4) with (j) confounding factors.

$$
\begin{gathered}
\text { IEHRP }=\left(\operatorname{IEHRI}_{1}, \operatorname{IEHRI}_{2}, \ldots, \operatorname{IEHRI}_{\mathrm{j}}\right) \\
=\left(\left[\mathrm{v}_{1,1}+\mathrm{v}_{1,2}+\mathrm{v}_{1,3}+\ldots+\mathrm{v}_{1, \mathrm{j}}\right],\left[\mathrm{v}_{2,1}+\mathrm{v}_{2,2}+\mathrm{v}_{2,3}+\ldots+\mathrm{v}_{2, \mathrm{j}}\right], \ldots,\left[\mathrm{v}_{\mathrm{i}, 1}+\mathrm{v}_{\mathrm{i}, 2}+\mathrm{v}_{\mathrm{i}, 3}+\ldots+\mathrm{v}_{\mathrm{i}, \mathrm{j}}\right]\right)
\end{gathered}
$$

With the development of the IEHRP for multiple exposures and multiple variables two key questions still needed to be addressed: (1) Which variable $\left(\mathrm{v}_{\mathrm{i}, \mathrm{j}}\right)$ was the most important? That is, should some weigh more than others? and; (2) How do we account for the variability of each variable?

To answer the first question (e.g. are genes more important than exposure measure?) the equation was enhanced with a "Weighting Factor" $\left(\mathrm{WF}_{\mathrm{i}, \mathrm{j}}\right)$, a numerical value that would account for importance of each variable $\left(v_{i, j}\right)$. To account for the variability (confidence) the equation included a "Correction Factor" $\left(\mathrm{CF}_{\mathrm{i}, \mathrm{j}}\right)$, a numerical value. Combining the CF with the WF resulted in the most recent iteration of the IEHRP, equation (5):

$$
\begin{aligned}
& \text { IEHRP }=\left(\text { IEHRI }_{1}, \text { IEHRI }_{2}, \ldots, \text { IEHRI }_{\mathrm{j}}\right) \\
& =\left(\left[\left(\mathrm{v}_{1,1}\right)\left(\mathrm{CF}_{1,1}\right)\left(\mathrm{WF}_{1,1}\right)+\left(\mathrm{v}_{1,2}\right)\left(\mathrm{CF}_{1,2}\right)\left(\mathrm{WF}_{1,2}\right)+\ldots+\left(\mathrm{v}_{1, \mathrm{j}}\right)\left(\mathrm{CF}_{1, \mathrm{j}}\right)\left(\mathrm{WF}_{1, \mathrm{j}}\right)\right]\right. \text {, } \\
& {\left[\left(\mathrm{v}_{2,1}\right)\left(\mathrm{CF}_{2,1}\right)\left(\mathrm{WF}_{2,1}\right)+\left(\mathrm{v}_{2,2}\right)\left(\mathrm{CF}_{2,2}\right)\left(\mathrm{WF}_{2,2}\right)+\ldots+\left(\mathrm{v}_{2, \mathrm{j}}\right)\left(\mathrm{CF}_{2, \mathrm{j}}\right)\left(\mathrm{WF}_{2, \mathrm{j}}\right)\right] \text {, }} \\
& \left.\left[\left(\mathrm{v}_{\mathrm{i}, 1}\right)\left(\mathrm{CF}_{\mathrm{i}, 1}\right)\left(\mathrm{WF}_{\mathrm{i}, 1}\right)+\left(\mathrm{v}_{\mathrm{i}, 2}\right)\left(\mathrm{CF}_{\mathrm{i}, 2}\right)\left(\mathrm{WF}_{\mathrm{i}, 2}\right)+\ldots+\left(\mathrm{v}_{\mathrm{i}, \mathrm{j}}\right)\left(\mathrm{CF}_{\mathrm{i}, \mathrm{j}}\right)\left(\mathrm{WF}_{\mathrm{i}, \mathrm{j}}\right)\right]\right)
\end{aligned}
$$

This IEHRP is for an individual, so if we imagine $\mathrm{N}$ individuals with similar or different exposures we would expect to see $\mathrm{N}$ distinct IEHRP's. Therefore, observing the collection of IEHRPs

$$
\left\{\operatorname{IEHRP}_{1}, \operatorname{IEHRP}_{2}, \ldots, \operatorname{IEHRP}_{\mathrm{N}}\right\}
$$

would be of interest.

For example, if we visualize the IHERPs for two individuals $\mathrm{N}=2$ (Figure 2), individual A has a high risk for noise whereas individual B has a high risk for radon. This visualization allows the provider or $\mathrm{IH} / \mathrm{OH}$ professional to target and prioritize interventions based on the individual's highest risk exposure(s). 


\section{IEHRP Benefits: Tailorability}
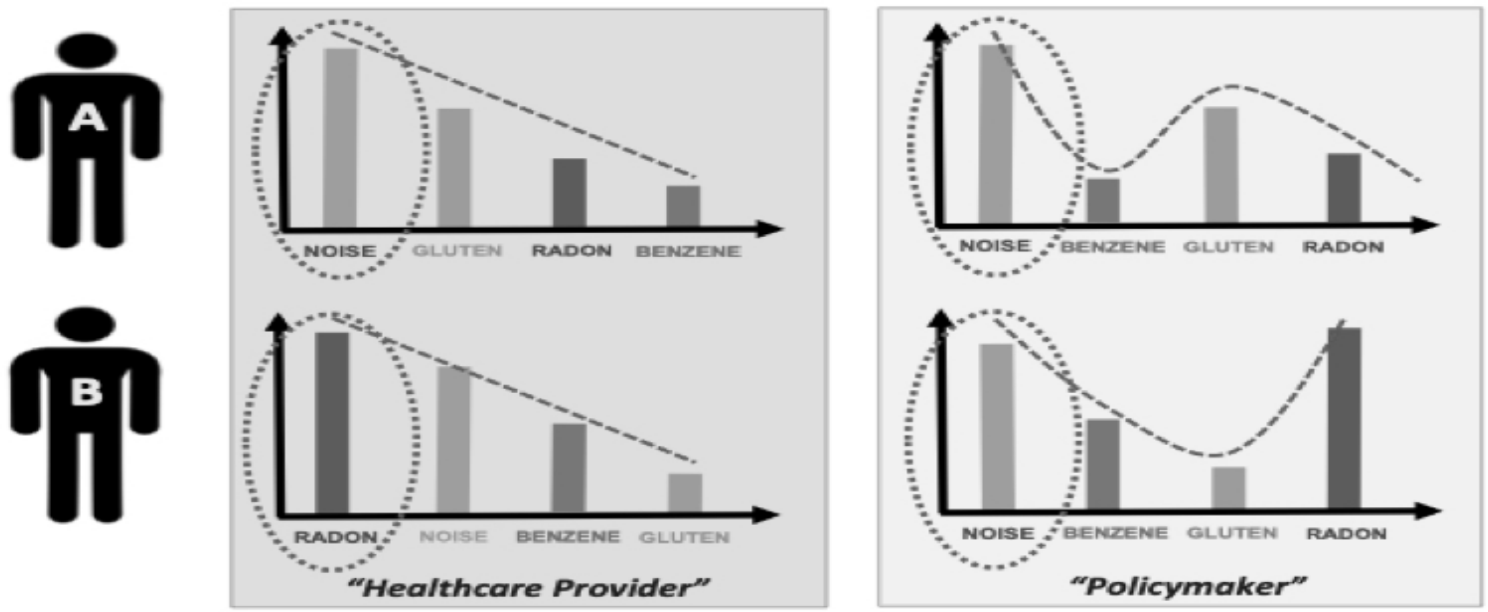

Figure 2. IHERP data visualization for personalized health and population health

If we modify the visualization with the same data to accommodate a policy maker, we notice noise is prevalent in both A and B, allowing the policy maker to identity the high priority exposure for either policy development/modification or proper resourcing versus focusing on a lower priority exposure.

When fully developed, the IEHRP will provide an enhanced capability to describe individual health risks based on genetic factors and occupational, lifestyle, and environmental exposure factors, medical disposition, protective factors, and other variables that affect exposure health risk.

\section{CONCLUSION}

Through initiatives like TEH we will uncover a new understanding of the relationships between an individual's genetic predispositions, epigenetic factors, and exposures from lifestyle, occupation, and the environment to support the development of diagnostic approaches, treatment methods, and intervention strategies to truly institute primary prevention to improve worker health, performance and productivity.

Through TEH, the healthcare profession will leverage and advance genomics, sensor and data technologies, data analytics, and health informatics into a future-focused and progressive approach that represents a disruptive but necessary paradigm to shift from a healthcare delivery system dependent heavily on infrastructure and staffing to one that allows individuals to take ownership of their health and drive their behaviors and health-related choices.

\section{REFERENCES}

Alamgir, H., D. L. Tucker, S.Y. Kim, et al.: Economic Burden of Hearing Loss for the U.S. Military: A Proposed Framework for Estimation. Military Medicine, Volume 181, Issue 4, 1 April 2016, Pages 301-306.

Carroll Y.I., E. J., Scinicariello, et al.: Vital Signs: Noise-Induced Hearing Loss Among Adults - United States 2011-2012. MMWR Morb Mortal Wkly Rep 2017;66:139-144.

National Institute for Occupational Safety and Health (NIOSH): “Total Worker Health." Available at https://www.cdc.gov/niosh/twh/default.html

PLOS ONE: "Genetic Polymorphisms Associated with Hearing Threshold Shift in Subjects during First Encounter with Occupational Impulse Noise," http://bit.ly/plosonenoise (June 2015). 